\title{
No Effect of Cooling on Cognitive Fatigue, Vigilance and Autonomic Functioning in Multiple Sclerosis
}

\author{
Anja Gossmann ${ }^{1}$, Paul Eling ${ }^{2}$, Andreas Kastrup ${ }^{1}$, and Helmut Hildebrandt ${ }^{1,3^{*}}$ \\ ${ }^{1}$ Klinikum Bremen-Ost, Department of Neurology, Züricher Str. 40, 28325 Bremen, Germany \\ ${ }^{2}$ Radboud University Nijmegen, Donders Institute for Brain, Cognition and Behaviour, Nijmegen, The Netherlands \\ ${ }^{3}$ University of Oldenburg, Institute of Psychology, 26111 Oldenburg, Germany
}

*Corresponding author: Helmut Hildebrandt, Department of Neurology, Klinikum Bremen-Ost, Züricher Str. 40, 28325 Bremen, Germany, Tel: 00494214081599; Fax: 00494214082599; E-mail: helmut-hildebrandt@uni-oldenburg.de

Received date: May 26, 2014, Accepted date: Aug 18, 2014, Published date: Aug 22, 2014

Copyright: (C) 2014 Hildebrandt $\mathrm{H}$, et al. This is an open-access article distributed under the terms of the Creative Commons Attribution License, which permits unrestricted use, distribution, and reproduction in any medium, provided the original author and source are credited.

\begin{abstract}
Background: Fatigue is a common symptom in Multiple Sclerosis (MS) and body cooling may be an important non-pharmacological treatment strategy for fatigue by, for instance, reducing the loss of axonal conduction efficiency due to Uhthoff's phenomenon. However, up to now, no studies have demonstrated such a treatment effect for mentally induced fatigue.

Methods: In this single-blinded randomized placebo controlled cross-over design we studied the effects of cooling on cognitive fatigue and autonomic functioning (heart rate variability and sympathovagal balance measures) during a vigilance task in $31 \mathrm{MS}$ patients and 10 controls.

Results: Task performance, fatigue and sympathovagal balance did not differ between verum- and placebo condition after controlling for depressive mood. MS patients showed more omissions on the vigilance test than controls; their performance declined during the task and this correlated significantly with cognitive fatigue. Cardiac sympathetic drive remained unchanged during vigilance testing in MS patients, but it increased significantly in controls.

Conclusion: Cooling has no impact on experienced cognitive fatigue and on cognitive performance in MS patients. Vigilance testing seems to be an appropriate behavioural measure of cognitive fatigue. Cardiac sympathetic drive to compensate for mental strain is reduced in MS patients, indicating an autonomic dysfunction.
\end{abstract}

Keywords: Multiple sclerosis; Fatigue; Autonomic functioning; Heart rate variability; Vigilance; Effects of cooling

\section{Introduction}

Fatigue is a common symptom in Multiple Sclerosis (MS) [1] Patients often report cognitive deficits in association with fatigue, but a clear connection between subjectively experienced cognitive limitations and objective performance on specific neuropsychological tests has not been demonstrated convincingly [2]. Moreover, the influence of fatigue on cognition has been assessed with subjective measures only in most studies. Hence, finding an adequate objective measure for fatigue is one of the aims of the present study.

Several explanations have been suggested for fatigue in MS [3] Most studies focused on supratentorial lesions or demyelination, but dysfunctions of the autonomic nervous system, have also been mentioned [4]. Autonomic dysfunctions like sleep disorders [5], loss of bladder control [6], depressive mood [7] and orthostatic hypotension are common in MS [8], statistically associated with fatigue [9]. A recent review argues for a direct link between hypothalamic lesions and fatigue in neurological diseases [10]. Presumably, a decrease of top-down control, due to demyelinated structures of the autonomic nervous system, results in a loss of bodily adaptation (i.e., changes in heart rate, blood pressure, distribution of cortisol and adrenaline) in reaction to mental and physical stress and thus to fatigue.

Demyelination of nerve fibres related to the motor system may play a direct role in increased fatigability of MS patients [3,10]. Uhthoff [11] was the first to observe an impaired acuity of MS patients after motor exhaustion. Later-on, exaggeration of neurological impairments was noticed in MS patients during fever, after taking a hot bath or visiting a sauna and it was termed the Uhthoff phenomenon $[12,13]$. The explanation for this exaggeration of symptoms is a relative loss of axonal conduction capacity in de- or incompletely remyelinated nerve fibres in response to heating of the body tissue.

Heat sensitivity is a common symptom in MS [14] and appears to be associated with fatigue [15] and cognitive performance [13]. Actually, increased sensitivity to heat may be a consequence of a loss of top-down autonomic nervous system control, as peripheral sudomotor activity and sweat gland innervations are controlled by brain stem areas and thermoregulation is under hypothalamic control. Reduced sweating and abnormal sympathetic skin responses have been reported in MS patients [14].

Given this relation between heat, conduction capacity of nerve fibres and fatigue, external cooling has been investigated as a treatment strategy. While there is some proof of amelioration of visual and motor symptoms $[16,17]$, to our knowledge no study examined 
the effect of cooling on performance and cognitive fatigue in mentally challenging tasks. We investigated the effect of a cooling vest in MS patients in a placebo controlled cross-over design. We used a vigilance test from the Test Battery for Attentional Performance (TAP) [18] for the following reasons. An older review showed that only performance in prolonged sustained attention tasks correlates with fatigue, [2] and a recent review on the cognitive signature of fatigue argues that vigilance and alertness are the only more experimental cognitive tasks that reliably correlate with fatigue in MS [20]. Alertness and vigilance rely on similar cognitive functions and neuroanatomical structures, [19] the latter being modulated by brain stem nuclei responsible for the release of noradrenaline and histamine, two neurotransmitter directly related to sleep-wake regulation. But only the vigilance task seems to induce cognitive fatigability directly correlated with experience of fatigue in MS patients, possibly due to its longer duration [2,20]. Moreover, vigilance performance correlates with heart rate variability (HRV) [21] and HRV seems to be a sensitive measure for autonomic nervous system activity and fatigue, as shown in cancer patients [22]. Neuroanatomical structures associated with vigilance are the locus coeruleus, the brain stem arousal system and right hemispheric frontal and parietal areas [23]. MS patients with fatigue show atrophy particularly in the right parietal and frontal cortex [24] and a vigilance decrement therefore can be expected.

This study aimed to examine whether fatigue is related to vigilance decrement and to reactivity of the autonomic nervous system, as measured by HRV, and whether a cooling vest reduces the increase of fatigue and of performance decline during such a cognitive task.

\section{Methods}

\section{Subjects}

Thirty-one patients with relapsing-remitting MS as defined by the McDonald criteria [25] and 10 controls participated (for demographic details, see table 1).

\begin{tabular}{|l|l|l|}
\hline & $\begin{array}{l}\text { MS Patients } \\
\text { Mean } \pm \mathrm{sd}\end{array}$ & $\begin{array}{l}\text { Healthy Controls } \\
\text { Mean } \pm \mathrm{sd}\end{array}$ \\
\hline Gender & $\mathrm{f}=26, \mathrm{~m}=5$ & $\mathrm{f}=6, \mathrm{~m}=4$ \\
\hline Age & $42.5 \pm 11.4$ & $42.8 \pm 10.6$ \\
\hline Disease duration & $124.7 \pm 110.2$ & - \\
\hline EDSS & $3.6 \pm 2.1$ & - \\
\hline FSMC & $72.8 \pm 17.5$ & $37.5 \pm 15.5$ \\
\hline BDI & $12.3 \pm 8.1$ & $3.8 \pm 4.0$ \\
\hline BDI-psy & $7.8 \pm 6.2$ & $2.2 \pm 2.6$ \\
\hline BDI-som & $5.2 \pm 4.1$ & $1.7 \pm 1.5$ \\
\hline
\end{tabular}

SD: Standard Deviation; EDSS: Expanded Kurtzke Disability Status Scale;

FSMC: Fatigue Scale for Motor and Cognitive Functions; BDI: Beck's Depression Inventory; psy: psychological component; som: somatic component

Table 1: Demographical and clinical characteristics of the groups.

All patients were relapse- and corticosteroid-free for at least six weeks prior to the study and without any psychiatric disorders or significant medical conditions (for example cardio-vascular dysfunctions, treatment with psychostimulants) that could confound the study. Patients were recruited by postings and circular letters inviting to participate in a study on cooling and fatigue. Healthy controls were selected mainly of the group of hospital's employees, trying to match for age, gender and education. The Expanded Disability Status Scale (EDSS) [26] served as a measure of disability. Approval was obtained from the Bremen Physicians Ethics Committee. All participants gave informed consent.

\section{Procedure}

Room temperature was held constant between 21 and 22 degrees Celsius, the normal house room temperature and participants adapted to this temperature during the first 25 minutes.

The cooling vest [27] was then prepared with 3 litres of cold water at $13^{\circ} \mathrm{C}$ for the verum condition, or 1 litre at $27^{\circ} \mathrm{C}$ for the placebocondition. The two conditions were presented in a counterbalanced order. Participants wore two thin cotton shirts during verum and 3 shirts plus an extra towel placed invisibly at the back during placebo condition. In both conditions the vest was worn for 40 minutes during vigilance testing with simultaneous collection of heart rate data and the fatigue assessment (visual analogue scale). There was a 20 minute break between conditions for re-adaptation to the room temperature.

We used a single-blind placebo controlled study design. Blinding of the patients was guaranteed by telling them that the main goal of the study is to find out the optimal cooling temperature for the vest, indicating that both cooling temperatures will work. The investigator was not blind for the conditions, but was unable to interfere with the PC based evaluation of the outcome measures.

\section{Assessments}

Body temperature was measured in-ear and on the patient's shoulder three times: at the beginning of the investigation as a baseline value and once after each cooling condition.

Vigilance testing was done for 30 minutes. The acoustic version was used because of possible visual impairments due to previous optic neuritis. Participants were instructed to listen to alternating beeps of 440 and $1000 \mathrm{~Hz}$, presented with an inter-stimulus interval of $1.3 \mathrm{sec}$. Occasionally, two beeps of the same frequency were presented in a row and the participants had to press a button as quickly as possible. There were 36 targets out of 1200 beeps over the whole test of 30 minutes. The interval between critical targets varied between 40 and 60 seconds. Omissions of target stimuli were calculated and time-on-task effects were determined by comparing omissions for the first 15 minutes and second 15 minutes. Before testing, participants practiced for 3 minutes to adapt to the beeps and the target sequence. We focused on omissions as they offer the most relevant information about drop of performance.

Participants were asked to rate their current feeling of fatigue on a $100 \mathrm{~mm}$ Visual Analog Scale (VAS, 0=no fatigue at all, 100=fully fatigued) at baseline and once during each cooling condition.

Heart rate as indication of autonomic nervous system activity was assessed during both cooling conditions, using the device "parport" from Par-Elektronik [28].

Electrodes were placed in standard positions under both clavicles and on the left side of the stomach. HR was assessed using the R-spike interval as the R-spike signal is easily detectable due to the high 
amplitude and the high flank steepness. Amplitude maximum was determined by a Schmitt-Trigger. The signal was standardized by a maximum detection which controlled a variable amplifier. The common mode rejection was performed by an initial high pass with $0.5-\mathrm{Hz}$ cut-off frequency. The $\mathrm{P}$ - and T-waves, which have a lower frequency spectrum than the R-spike, were rejected by a band pass with cut-off frequencies of 20 and $30 \mathrm{~Hz}$. In addition, an R-spike interval lower than $240 \mathrm{~ms}$ was prevented by a monostable multivibrator. So the maximum HR could be $250 \mathrm{~min}-1$. Heart rate was converted by Fast Fourier Transformation (FFT) and a timedependent spectral analysis was performed. According to the consensus statement on HRV analysis (1996), [29] band width was defined as between $0.012 \mathrm{~Hz}$ to $0.043 \mathrm{~Hz}$ for the very low-frequency band, $0.047 \mathrm{~Hz}$ to $0.152 \mathrm{~Hz}$ for the low-frequency band and $0.156 \mathrm{~Hz}$ to $0.402 \mathrm{~Hz}$ for the high-frequency band. After log transformation of these data, we calculated the ratio of very low frequency to high frequency $(\mathrm{VLF} / \mathrm{HF})$ and the ratio of low frequency to high frequency (LF/HF) as estimates of sympathetic activity and sympathovagal balance.

The beginning and end of the vigilance test was marked electronically. Physiological data were inspected for technical artifacts and time-on-task effects were analyzed by averaging the results of the first and second 15 minutes period.

Additionally, participants completed the Fatigue Scale for Motor and Cognitive Functions (FSMC) [30]. According to the manual of the FSMC the cut-off score of 43 was used to divide the MS patients in those with and without fatigue. We also presented the Beck's Depression Inventory (BDI) [31] and determined the psychological component and the somatic component of depressive mood separately by adding items A-L for the first and M-U for the latter aspect. The somatic items also concern sleepiness and sleep impairments. Hence, we focused on the psychological items to correct for depressive mood in the statistical evaluations.

\section{Statistical evaluation}

Repeated measures analyses of variance (ANCOVA) were used to analyse performance on the vigilance task. To control for a potential effect of depressive symptoms on fatigue, the score on the psychological component of BDI was used as a covariate in all analyses.

Verum versus Placebo was defined as the within-group factor Treatment. Time-on-task effects were defined as the within-group factor Time (first half against second half of the 30 minutes period of vigilance testing). The between subjects factor was defined as Group (patients vs controls). Significant interactions between Group and Treatment and Group and Time were further analysed by t-tests for dependent measurements.

We first compared the whole MS group with the healthy controls and subsequently repeated the analysis excluding MS patients without fatigue $(n=3)$.

\section{Results}

\section{Effect of cooling on body temperature}

Cooling led to a highly significant difference between the Verum and Placebo condition (main effect Treatment [F (1,38): 88.696, $\mathrm{p}<0.001]$, a main effect of Place (skin versus ear)

[F $(1,38): 34.870, p<0.001)]$, but there was no main effect of Group and there were no significant interaction effects. Decline of temperature was much higher when measured at the shoulder than in the ear (Table 2). Excluding MS patients without fatigue the ANCOVA produced similar statistical results.

\begin{tabular}{|c|c|c|c|c|c|c|}
\hline & \multicolumn{2}{|c|}{ Initial measurement } & \multicolumn{2}{|l|}{ Verum } & \multicolumn{2}{|l|}{ Placebo } \\
\hline & MS & Controls & MS & Controls & MS & Controls \\
\hline Visual analogue scale (Fatigue) & $29.2 \pm 22.3$ & $29.4 \pm 25.8$ & $40.7 \pm 26.7^{*}$ & $42.6 \pm 25.1$ & $51.3 \pm 28.1^{*}$ & $48.1 \pm 19.7$ \\
\hline Temperature Ear & $37.0 \pm 0.4$ & $36.8 \pm 0.4$ & $36.9 \pm 0.4$ * & $36.8 \pm 0.4$ * & $36.9 \pm 0.4$ * & $36.8 \pm 0.5$ \\
\hline Skin & $34.5 \pm 1.4$ & $34.6 \pm 1.1$ & $32.0 \pm 1.8^{*}$ & $32.1 \pm 2.6^{*}$ & $33.7 \pm 1.8^{*}, \#$ & $34.0 \pm 2.1^{*}, \#$ \\
\hline Heart rate (variance) & & & $2.5 \pm 1.0$ & $2.5 \pm 1.0$ & $2.5 \pm 0.5$ & $2.6 \pm 0.7$ \\
\hline
\end{tabular}

Table 2: Effects of external cooling.

\section{Fatigue}

Three MS patients showed no fatigue according to the cut-off score of the MSFC. MS patients with fatigue scored significantly higher on FSMC than controls $(\mathrm{p}<0.001)$. Also, the psychological component of the BDI was significantly higher among MS patients $(\mathrm{p}=0.004)$. The two groups did not differ in age, education and gender.

The application of cooling (Treatment) had no significant impact on fatigue [F $(1,38): 3.178, p=0.054]$ as measured with the visual analogue scale, but there was a significant Group difference $[\mathrm{F}(1,38)$ :
6.820, $\mathrm{p}=0.003$ ]. The interaction between Group and Treatment was not significant. Patients without cognitive fatigue showed the lowest fatigue score, patients with fatigue the highest and healthy controls scored in between these two groups.

T-tests for dependent measures for MS patients, not controlling for depressive mood, showed significant differences between cognitive fatigue scores before and after vigilance testing in the Verum condition, and there was also a significant difference between verum and placebo condition. No difference between verum and placebo was 
found for the healthy controls, and this might be due to the small number of participants (Table 2).

Hence, fatigue increased during the vigilance task in both groups (significant dependent t-tests), which was performed just before the fatigue measurement in the Placebo and the Verum condition. Fatigue was also higher for MS patients in the Verum than in the Placebo condition, but this effect disappeared when we controlled for depressive mood (no significant interaction effect between Group and Treatment in the ANCOVA).

\section{Vigilance}

The ANCOVA of the vigilance task revealed a main effect of Time [F $(1,38): 6.102, p=0.018]$, a main effect of Group [F (1,38): 5.801, $\mathrm{p}=0.021]$, and a significant interaction between Time and Group [ $\mathrm{F}$ (1,38): 6.096, $\mathrm{p}=0.018]$. There was no main effect of Treatment or a significant interaction between Treatment and any of the other factors.

Excluding MS patients without fatigue we found a main effect of Time [F (1,35): 8.460, $\mathrm{p}=0.006]$, a main effect of Group [F $(1,35)$ : $8.555, \mathrm{p}=0.006]$, and a significant interaction between Time and Group [F (1,35): 8.296, $\mathrm{p}=0.007]$. MS Patients with fatigue made more omissions than healthy controls irrespective of cooling condition. Moreover, patient's performance declined significantly during the task (Table 2), while healthy controls improved slightly (Figure 1). Therefore, the application of active temperature cooling (verum condition) did not improve performance of MS patients in comparison to that of healthy controls, but MS patients performed worse in the vigilance test and were more sensitive to time-on-task in the vigilance test.

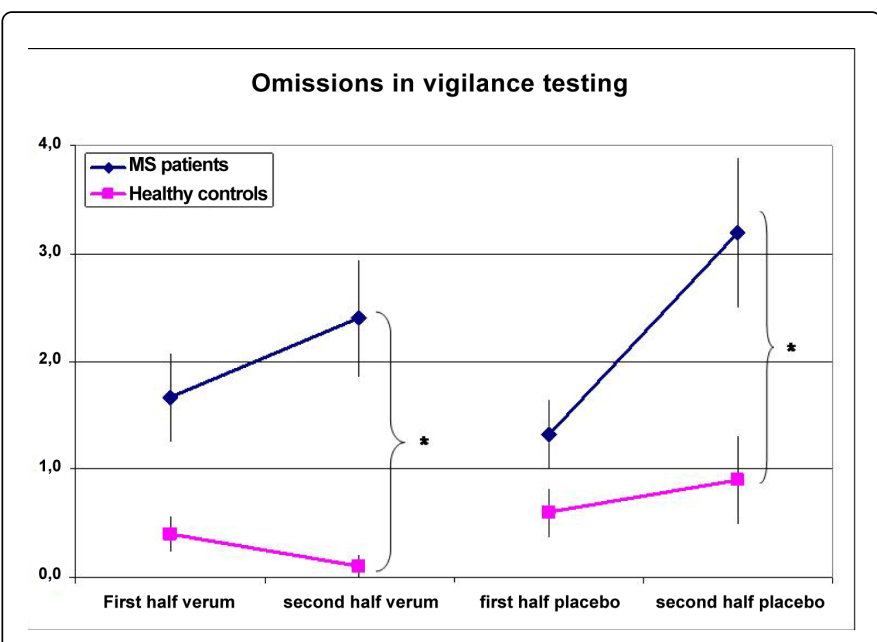

Figure 1: Omissions in vigilance testing.

Error bars: standard error of mean; ${ }^{*} \mathrm{p}<0.05$ for independent t-test.

To check whether there was a pure sequence between the conditions (for instance, a decrement of performance in condition two because having already performed condition one), we rearranged our data and did the same statistical analyses as mentioned above. Because the sequence factor did not yield any significant results, we can exclude such an influence on our data.

\section{HRV analysis}

Data from 5 participants showed technical artifacts during one of the measurements and were excluded from the analysis.

Comparing the whole group of MS patients with healthy controls on the LF/HF ratio, there was no main effect of Treatment, Time and Group, but there was a significant interaction between Time and Group [F (1,33): 5.095, p=0.031], showing a small decrease of LF/HF in MS patients while the scores of controls increased over time.

Comparing only MS patients with fatigue to healthy controls, there was no main effect of Treatment, Time or Group, but there was a significant interaction between Time and Group [F (1,30): 4.599, $\mathrm{p}=0.04]$. Dependent $\mathrm{t}$-tests did not reveal significant differences.

Comparing the whole group of MS patients with healthy controls, the ANCOVA on the VLF/HF ratio showed no main effect of Treatment, Time and Group, and no significant interactions. The main effect of Group approached significance $(\mathrm{p}=0.076)$ (Figure 3).

Comparing only MS patients with fatigue to healthy controls did not change these results.

Hence, vigilance testing and not cooling affected heart rate variability differentially in MS patients and healthy controls.

\section{Post-hoc correlational analyses (only for MS patients)}

The EDSS scores did not correlate with performance on the vigilance tasks (split up for the first and second half of the task) and the VAS scores. Therefore, our results cannot simply be explained by the degree of disability.

Fatigue as measured with a visual analogue scale, before and after each vigilance task, correlated with the number of omissions made in the second half of the vigilance test, even when controlling for the sum score of psychological items of the BDI (Table 3), but it did not correlate with HRV.

\section{Discussion}

External cooling did not result in a subjective or objective amelioration of fatigue that differed from that in the placebo condition, but we found a fatigue-related performance decline on a vigilance test and a reduced reaction in cardiac sympathetic drive in response to vigilance testing in MS patients compared to healthy controls.

Wearing a cooling vest neither led to a reduction of fatigue (at least not after controlling for depressive mood) nor did it improve performance on the vigilance task. This deviates from findings of some previous studies [32-35]. Several aspects have to be mentioned, which might explain our negative results. First of all, none of these previous studies controlled statistically for the effect of depressive mood. Actually, without such a control our patients would have experienced significantly less fatigue in the verum condition compared to the placebo condition, which was our original expectation (Table 2). Secondly, we focused on cognitive fatigue and cognitive performance. The only study we are aware of that measured cognitive performance in MS patients with fatigue (but not specifically cognitive fatigue) before and after cooling revealed - as our study did - no improvement in cognition, but in motor efficiency [36]. Motor efficiency, measured by timed up and go test and timed walking tests, was often used to 
measure improvement after cooling [37,38]. Therefore, one explanation could be that cooling affects motor performance.
Accordingly, non-motor functions like visual acuity, which also has been assessed in previous studies, did not response to cooling $[36,39]$.

\begin{tabular}{|l|l|l|l|l|l|l|l|l|l|l|l|l|}
\hline & \multicolumn{2}{l}{ Verum, 1st half } & \multicolumn{2}{l|}{ Verum, 2nd half } & \multicolumn{2}{l|}{ Placebo, 1st half } & \multicolumn{2}{l|}{ Placebo, 2nd half } \\
\hline & MS & Controls & $\mathrm{p}$ & MS & Controls & $\mathrm{p}$ & MS & Control & $\mathrm{p}$ & $\mathrm{MS}$ & Control & $\mathrm{p}$ \\
\hline $\begin{array}{l}\text { Vigilance } \\
\text { (omissions) }\end{array}$ & $1.7 \pm 2.3$ & $0.4 \pm 0.5$ & 0.007 & $2.4 \pm 2.3$ & $0.1 \pm 0.3$ & 0.000 & $1.3 \pm 1.7$ & $0.6 \pm 0.7$ & 0.067 & $3.2 \pm 3.9$ & $0.9 \pm 1.3$ & 0.007 \\
\hline LF/HF & $0.8 \pm 0.3$ & $1.0 \pm 0.3$ & 0.188 & $0.8 \pm 0.4$ & $1.1 \pm 0.2$ & 0.049 & $0.9 \pm 0.3$ & $1.0 \pm 0.2$ & 0.353 & $0.9 \pm 0.3$ & $1.0 \pm 0.2$ & 0.107 \\
\hline VLF/HF & $1.0 \pm 0.4$ & $1.2 \pm 0.5$ & 0.261 & $0.9 \pm 0.5$ & $1.3 \pm 0.3$ & 0.044 & $1.0 \pm 0.4$ & $1.2 \pm 0.2$ & 0.080 & $1.0 \pm 0.4$ & $1.2 \pm 0.3$ & 0.200 \\
\hline
\end{tabular}

p: Significance level for independent testing; LF/HF: ratio low frequency/ high frequency components; VLF/HF: very low frequency/ high frequency components, Mean \pm Standard deviation

Table 3: Effects of external cooling.

Second, most of the studies examined only heat sensitive patients with fatigue; heat sensitivity was not an inclusion criterion in our study $[39,40]$. Heat sensitivity is one aspect of fatigue, but is not experienced by all MS patients with fatigue. Perhaps, only heat sensitive MS patients profit from cooling. Sumowski and Leavitt found that body temperature is closely related to motor fatigue but not to cognitive fatigue [41].

Third, differences in the effects of cooling procedures among studies may also play a role. Inner ear temperature declined only marginally in our MS patients group $\left(0.1^{\circ} \mathrm{C}\right)$. Previous studies reported declines of inner ear temperature of $0.3^{\circ} \mathrm{C}$ to $0.4^{\circ} \mathrm{C}$, but this was not the case for all studies [42]. These differences between the studies are remarkable and not easy to explain. We used a cooling vest, a cooling temperature far below the value that is proposed by the manufacturer and a cooling period of 30 minutes. This is similar to procedures from previous studies, and many of the MS patients indicated to suffer from wearing the vest with such a low temperature. The subjectively experienced cooling was so intense that a more rigorous cooling would exclude any clinical application. Last but not least, our cooling resulted in a significant difference between placebo- and verum-condition, and skin temperature decreased after verum cooling about $2.5^{\circ} \mathrm{C}$ in $\mathrm{MS}$ patients and only $0.7^{\circ} \mathrm{C}$ after placebo cooling (compared to skin temperature before cooling).

Hence, cooling may be effective for motor, but not for cognitive fatigue, because exhausting motor performance leads to increase of body heat and demyelinated nerve fibres then lose their ability of efficient conduction relative to tissue temperature (the Uhthoff phenomenon) [13], a mechanism that is irrelevant during cognitive processing. On the other hand, we cannot exclude that more effective cooling, as in our study, may lead to improvement in cognitive tasks, too.

Vigilance testing clearly induced objective fatigue, i.e. the typical vigilance decrement [19] in MS patients (Figure 1). Moreover, compared to the healthy control participients subjects, sympathetic heart rate modulation did not increase (Figure 2 and 3 ).

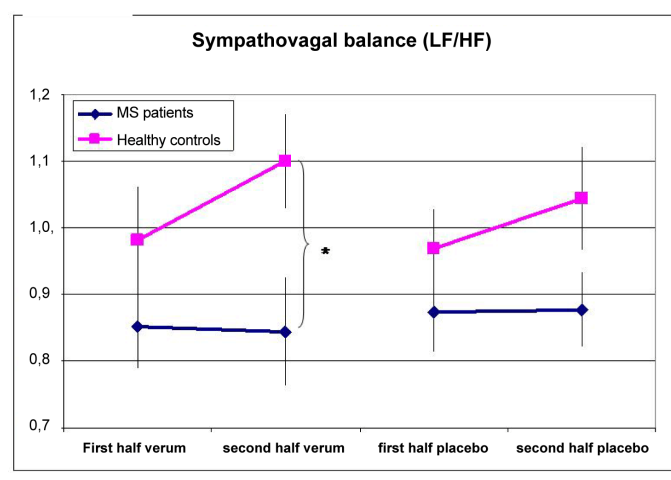

Abbreviations: $L F=$ low frequency heart rate variability $(0.047 \mathrm{~Hz}$ to $0.152 \mathrm{~Hz})$ $\mathrm{HF}=$ high frequency of heart rate variablity $(0.156 \mathrm{~Hz} 0.402 \mathrm{~Hz})$ Error bars: standard error of mean; ${ }^{*} P<0.05$ for independent t-test

Figure 2: Sympathovagal balance (LF/HF).

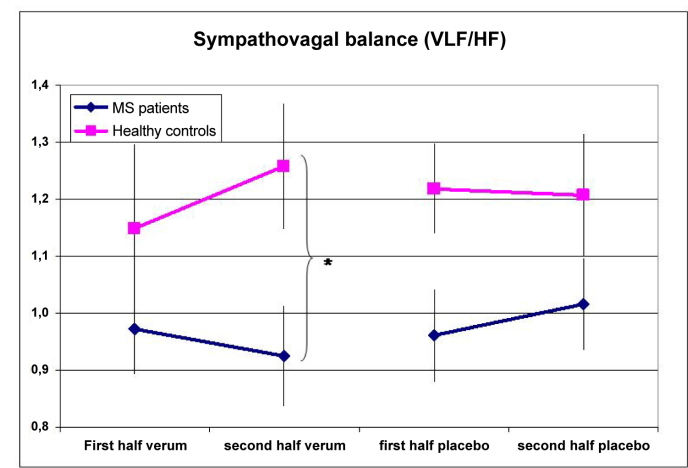

Abbreviations: VLF= very low frequency heart rate variability $(0.012 \mathrm{~Hz}$ to $0.043 \mathrm{~Hz})$ $\mathrm{HF}=$ high frequency of heart rate variablity $(0.156 \mathrm{~Hz} 0.402 \mathrm{~Hz})$ Error bars: standard error of mean; ${ }^{*} \mathrm{P}<0.05$ for independent $\mathrm{t}$-test

Figure 3: Sympathovagal balance (VLF/HF). 
In his excellent review DeLuca [2] distinguished between cognitive fatigue induction by extended periods of mental or motor testing or by sustained attention tasks. Simply challenging patients with tasks over an extended period of time did not produce task related impairments. On the other hand, studies using sustained attention tasks did find a decrease in performance. For instance, performance on an alertness task, but not on memory tests, correlates with fatigue [20]. Unfortunately, the alertness task shows a decrease of objective performance in MS patients only after an extended period of exhaustion. Moreover, it does not correlate with the increase of fatigue during that performance [20]. In our investigation differences between patients and controls became evident on the vigilance task already after 15 minutes, and performance decline correlated with experienced cognitive fatigue (Table 3 ). Therefore the vigilance test appears to be a well-suited measure for cognitive fatigue.

As a biological marker of increased fatigability we also found a HRV dysregulation in MS patients performing the vigilance task (Figure 2 and Figure 3). This is in line with other studies demonstrating a role of the autonomic nervous system in the development of fatigue in reaction to mental strain [34] or to postural changes [33]. The difference between patients and controls was more evident for the LF/HF than for the VLF/HF ratio. Although both measures are considered to be indicators of sympathovagal balance, the assessment of $\mathrm{VLF} / \mathrm{HF}$ is not recommended for short-term recordings in the consensus statement of 1996 [29] as the physiological interpretation of this component is not clear. This might explain the restricted effect of the vigilance task on this latter ratio. The reduced sympatheticautonomic response to cognitive load in MS patients supports the hypothesis of a lack of cortical top-down control due to either demyelinization of fibres in the brain stem or damage to subcortical (hypothalamic) areas. However, the LF/HF and VLF/HF scores did neither correlate with test performance nor with fatigue. Therefore, future studies have to show whether and how HRV dysregulation and increased feeling of fatigue may be related.

Our study has some limitations. First, to ensure blinding we decided that some degree of cooling was necessary in the placebo condition, which may have had a minor effect on the results. Furthermore, including more MS patients without fatigue would have been helpful in order to distinguish between fatigue and other MSrelated symptoms. A strong point was that we assessed cognitive performance, experienced cognitive fatigue and physiological parameters of fatigue. Moreover, potential influences of depressive mood on vigilance were statistically controlled for. As a clinically relevant finding, our data indicate that fatigue should be assessed by looking for vigilance decrements. Moreover, autonomic dysfunctions may be more common in MS patients if heart rate variability is measured under conditions of sustained attention over periods longer than thirty minutes. On the other hand, according to our data, cooling is ineffective in ameliorating subjectively and objectively measured cognitive fatigue.

\section{References}

1. Krupp LB, Serafin DJ, Christodoulou C (2010) Multiple sclerosisassociated fatigue. Expert Rev Neurother 10: 1437-1447.

2. DeLuca J (2005) Fatigue, cognition, and mental effort. In Fatigue as a window to the brain Cambridge: Mit press, pp: 37-57.

3. Kos D, Kerckhofs E, Nagels G, D'hooghe MB, Ilsbroukx S (2008) Origin of fatigue in multiple sclerosis: review of the literature. Neurorehabil Neural Repair 22: 91-100.
4. Merkelbach S, Haensch CA, Hemmer B, Koehler J, König NH, et al. (2006) Multiple sclerosis and the autonomic nervous system. J Neurol 253 Suppl 1: I21-25.

5. Veauthier C, Radbruch H, Gaede G, Pfueller CF, Dörr J, et al. (2011) Fatigue in multiple sclerosis is closely related to sleep disorders: a polysomnographic cross-sectional study. Mult Scler 17: 613-622.

6. Dallmeijer AJ, Dekker J, Roorda LD, Knol DL, van Baalen B, et al. (2005) Differential item functioning of the Functional Independence Measure in higher performing neurological patients. J Rehabil Med 37: 346-352.

7. Brown RF, Valpiani EM, Tennant CC, Dunn SM, Sharrock M, et al. (2009) Longitudinal assessment of anxiety, depression, and fatigue in people with multiple sclerosis. Psychol Psychother 82: 41-56.

8. Flachenecker P, Wolf A, Krauser M, Hartung HP, Reiners K (1999) Cardiovascular autonomic dysfunction in multiple sclerosis: correlation with orthostatic intolerance. J Neurol 246: 578-586.

9. Kale N, Magana S, Agaoglu J, Tanik O (2009) Assessment of autonomic nervous system dysfunction in multiple sclerosis and association with clinical disability. Neurol Int 1: e5.

10. Kluger BM, Krupp LB, Enoka RM (2013) Fatigue and fatigability in neurologic illnesses: proposal for a unified taxonomy. Neurology 80: 409-416.

11. Uhthoff $\mathrm{W}$ (1890) Untersuchungen über die bei der multiplen herdsklerose vorkommenden augenstörungen. Arch Psychiatr Nervenkrankh 21: 305-410.

12. Guthrie TC, Nelson DA (1995) Influence of temperature changes on multiple sclerosis: critical review of mechanisms and research potential. J Neurol Sci 129: 1-8.

13. Marino FE (2009) Heat reactions in multiple sclerosis: an overlooked paradigm in the study of comparative fatigue. Int J Hyperthermia 25: 34-40.

14. Davis SL, Wilson TE, White AT, Frohman EM (2010) Thermoregulation in multiple sclerosis. J Appl Physiol (1985) 109: 1531-1537.

15. Flensner G, Ek AC, Söderhamn O, Landtblom AM (2011) Sensitivity to heat in MS patients: a factor strongly influencing symptomology--an explorative survey. BMC Neurol 11:27.

16. Schwid SR, Petrie MD, Murray R, Leitch J, Bowen J, et al. (2003) A randomized controlled study of the acute and chronic effects of cooling therapy for MS. Neurology 60: 1955-1960.

17. White AT, Wilson TE, Davis SL, Petajan JH (2000) Effect of precooling on physical performance in multiple sclerosis. Mult Scler 6: 176-180.

18. Zimmermann PF, Testbatterie B (1992) zur aufmerksamkeitsprüfung. Herzogenrath: Psytest.

19. Parasuraman RW, J S, See JE (1998) Brain systems of vigilance. In Parasuraman (ed) The attentive brain, Mit press, Cambridge, pp221-256.

20. Hanken KE, Hildebrandt $\mathrm{H}$ (2014) Is there a cognitive signature of ms related fatigue?

21. Chua EC, Tan WQ, Yeo SC, Lau P, Lee I, et al. (2012) Heart rate variability can be used to estimate sleepiness-related decrements in psychomotor vigilance during total sleep deprivation. Sleep 35: 325-334.

22. Fagundes CP, Murray DM, Hwang BS, Gouin JP, Thayer JF et al. (2011) Sympathetic and parasympathetic activity in cancer-related fatigue: More evidence for a physiological substrate in cancer survivors. Psychoneuroendocrinology. 36: 1137-1147.

23. Paus $\mathrm{T}$ (2000) Functional anatomy of arousal and attention systems in the human brain. Prog Brain Res 126: 65-77.

24. Calabrese M, Rinaldi F, Grossi P, Mattisi I, Bernardi V, et al. (2010) Basal ganglia and frontal/parietal cortical atrophy is associated with fatigue in relapsing-remitting multiple sclerosis. Mult Scler 16: 1220-1228.

25. McDonald WI, Compston A, Edan G, Goodkin D, Hartung HP, et al. (2001) Recommended diagnostic criteria for multiple sclerosis: Guidelines from the international panel on the diagnosis of multiple sclerosis. Ann Neurol. 50: 121-127.

26. Kurtzke JF (1983) Rating neurologic impairment in multiple sclerosis: an expanded disability status scale (EDSS). Neurology 33: 1444-1452. 
Citation: Gossmann A, Eling P, Kastrup A, Hildebrandt H (2014) No Effect of Cooling on Cognitive Fatigue, Vigilance and Autonomic Functioning in Multiple Sclerosis. J Mult Scler 1: 112. doi:10.4172/jmso.1000112

Page 7 of 7

27. http://www.E-cooline.De/produkte/classic-vest.2012

28. http://www.Par-berlin.Com/par_port_f.htm.2013

29. Heart rate variability (1996) Standards of measurement, physiological interpretation, and clinical use. Task force of the european society of cardiology and the north american society of pacing and electrophysiology. Eur Heart J 17: 354-381.

30. Penner IK, Raselli C, Stöcklin M, Opwis K, Kappos L, et al. (2009) The Fatigue Scale for Motor and Cognitive Functions (FSMC): validation of a new instrument to assess multiple sclerosis-related fatigue. Mult Scler 15: 1509-1517.

31. Beck ATH, Bailer M (1985). Bdi-beck-depressions-inventar. Hogrefe, göttingen.

32. Meyer-Heim A, Rothmaier M, Weder M, Kool J, Schenk P (2007) Advanced lightweight cooling-garment technology: Functional improvements in thermosensitive patients with multiple sclerosis. Mult Scler, 13: 232-237.

33. Monge-Argilés JA, Palacios-Ortega F, Vila-Sobrino JA, Matias-Guiu J (1998) Heart rate variability in multiple sclerosis during a stable phase. Acta Neurol Scand 97: 86-92.

34. Capello E, Gardella M, Leandri M, Abbruzzese G, Minatel C, et al. (1995) Lowering body temperature with a cooling suit as symptomatic treatment for thermosensitive multiple sclerosis patients. Ital J Neurol Sci 16: 533-539.

35. Flensner G, Lindencrona C (2002) The cooling-suit: case studies of its influence on fatigue among eight individuals with multiple sclerosis. J Adv Nurs 37: 541-550.
36. Geisler MW, G EA, Squires NK, Coyle PK, Doscher C, et al. (1996) Cooling and multiple sclerosis: Cognitive and sensory effects. Neurorehabil Neural Repair 10: 17-21.

37. Grahn DA, Murray JV, Heller HC (2008) Cooling via one hand improves physical performance in heat-sensitive individuals with multiple sclerosis: a preliminary study. BMC Neurol 8: 14.

38. Schwid SR, Petrie MD, Murray R, Leitch J, Bowen J, et al. (2003) A randomized controlled study of the acute and chronic effects of cooling therapy for MS. Neurology 60: 1955-1960.

39. Reynolds LF, Short CA, Westwood DA, Cheung SS (2011) Head precooling improves symptoms of heat-sensitive multiple sclerosis patients. Can J Neurol Sci 38: 106-111.

40. Nilsagård Y, Denison E, Gunnarsson LG (2006) Evaluation of a single session with cooling garment for persons with multiple sclerosis--a randomized trial. Disabil Rehabil Assist Technol 1: 225-233.

41. Sumowski JF, Leavitt VM2 (2014) Body temperature is elevated and linked to fatigue in relapsing-remitting multiple sclerosis, even without heat exposure. Arch Phys Med Rehabil 95: 1298-1302.

42. Beenakker EA, Oparina TI, Hartgring A, Teelken A, Arutjunyan AV, et al. (2001) Cooling garment treatment in MS: clinical improvement and decrease in leukocyte NO production. Neurology 57: 892-894. 